\title{
General population growth models with Allee effects in a random environment
}

\author{
Clara Carlos* \\ Escola Superior de Tecnologia do Barreiro, Instituto Politécnico de Setúbal, Rua Américo \\ da Silva Marinho, 2839-001 Lavradio, Portugal \\ Centro de Investigação em Matemática e Aplicações, Instituto de Investigação e Formação \\ Avançada, Universidade de Évora, Rua Romão Ramalho, 59, 7000-671 Évora, Portugal \\ Carlos A. Braumann ${ }^{1, *}$ \\ Departamento de Matemática, Escola de Ciências e Tecnologia, Universidade de Évora, \\ Rua Romão Ramalho, 59, 7000-671 Évora, Portugal \\ Centro de Investigação em Matemática e Aplicações, Instituto de Investigação e Formação \\ Avançada, Universidade de Évora, Rua Romão Ramalho, 59, 7000-671 Évora, Portugal
}

\begin{abstract}
Allee effects on population growth are quite common in nature, usually studied through deterministic models with a specific growth rate function.

In order to seek the qualitative behaviour of populations induced by such effects, one should avoid model-specific behaviours. So, we use as a basis a general deterministic model, i.e. a model with a general growth rate function, to which we add the effect on the growth rate of the random fluctuations in environmental conditions. The resulting model is the general stochastic differential equation (SDE) model that we propose here.
\end{abstract}

We consider two possible cases, weak Allee effects and strong Allee effects, which lead to different qualitative behaviours of the model.

We will study the model properties for both cases in terms of existence and uniqueness of the solution, extinction and stationary behaviour of the population. The two cases will be compared with each other and with the general

\footnotetext{
* Corresponding author

Email address: clara.carlos@estbarreiro.ips.pt (Clara Carlos)

${ }^{1}$ braumann@uevora.pt
} 
density-dependent SDE model without Allee effects.

We then consider as an example the particular case of the classic logistic model and an Allee effect version of it.

Keywords: Allee effects, Population growth, Random environments, Extinction times

2010 MSC: 92D25, 60H10

\section{Introduction}

Let $X(t)$ be the population size at time $t \geq 0$ and $f(X)$ the per capita growth rate when the population has size $X$. Assume the initial population size $X(0)=x>0$ is known.

$5 \quad$ Allee effects, named after Warder Clyde Allee, are exhibited by some populations when its density $X$ is low due, for instance, to the difficulty of finding mating partners or the inefficiency of group defence against predators. When Allee effects are absent, we expect $f$ to be a decreasing function of population size. When, however, such effects are present, we witness an unexpected behaviour of $f$, namely its increase with $X$ at low densities (see [1]), when the Allee effects are felt by the population, while at higher densities $f$ still follows the expected decreasing behaviour. There are two kind of Allee effects, strong Allee effects (when the growth rate is even negative for very low densities) and weak Allee effects (when the growth rate, although depressed, is still positive for low densities).

In this work we consider environmental stochasticity, i.e. we assume the environment is subjected to random fluctuations that affect the growth rate. So, we will use as a basis a deterministic model to which we add the effect on the growth rate of such environmental fluctuations.

Deterministic models of Allee effects have been proposed in the literature (see, for instance, 2, 3] and [4]), but they consider specific forms for the per capita growth rate function $f$. A commonly used model, which we illustrate on Figure 1, is a variation of the classical logistic model. However, no one knows the 

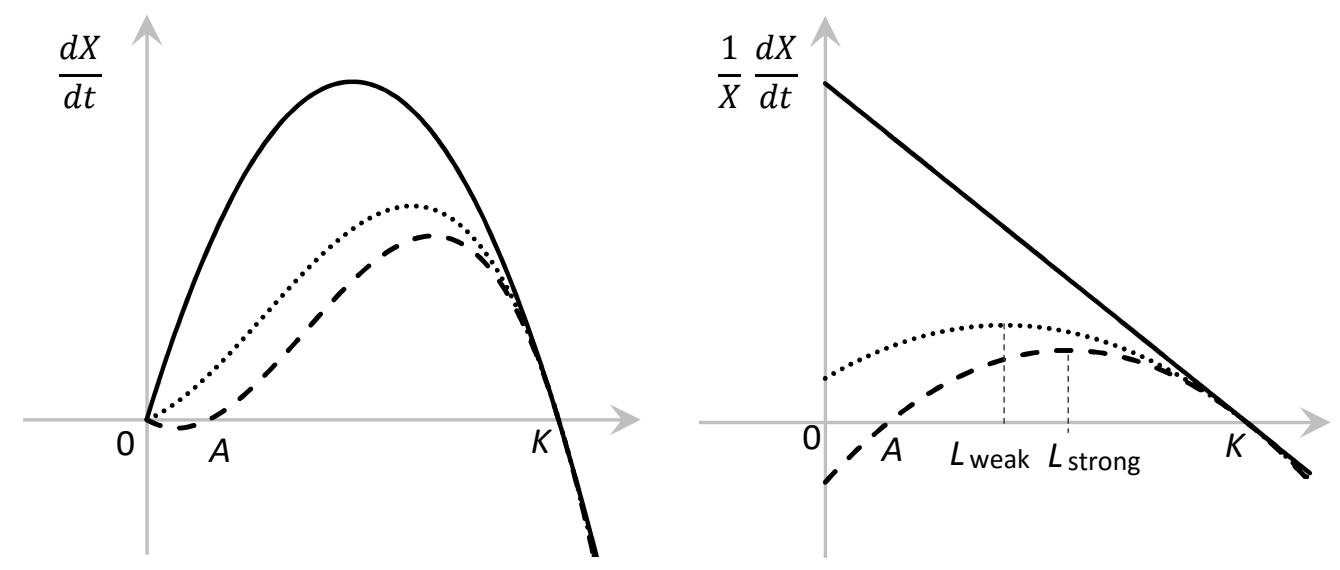

Figure 1: Example (logistic or logistic-like deterministic models) of the (per capita) growth rate $\frac{1}{X} \frac{d X}{d t}$ (on the left) and the total population growth rate $d X / d t$ (on the right) as a function of population size. The solid line represents a model with no Allee effects, the dashed line represents a model with strong Allee effects and the dotted line represents a model with weak Allee effects.

specific form that $f$ really takes and so we want to obtain properties concerning the qualitative behaviour of populations subjected to Allee effects that are not model-specific. We rather seek for properties that are robust with respect to model choice. So, we are going to consider as a basis a general deterministic model of the form

$$
\frac{1}{X(t)} \frac{d X(t)}{d t}=f(X(t)), \quad X(0)=x>0
$$

where the per capita growth rate $f$ is any function satisfying appropriate conditions dictated by biological considerations. We will also assume from now on that $f(X)$, defined for $X>0$, satisfies some mild technical conditions, namely that it is a function of class $C^{1}$ such that $f\left(0^{+}\right)$is finite and different from zero.

Let us look at the biologically dictated assumptions. 
Whether or not there are Allee effects, the environment cannot sustain an infinitely large population. So, $f$ should have negative values for very large population sizes since then resources would be insufficient to keep the birth rate above the death rate. We therefore assume from now on that $f(+\infty)<0$.

The above assumptions are common to all cases, whether there are Allee effects, either strong or weak, or not. Let us now look at assumptions that depend on the case we are considering.

If there are no Allee effects, one expects that, as the population size $X$ gets larger, the resources available for an individual to survive and reproduce become shorter and therefore the per capita growth rate $f(X)$ should be a strictly decreasing function of $X$. This, of course, will fail for low population 45 sizes if there are Allee effects since other factors (like for instance, inefficiency of group defence against predators or difficulty in finding mating partners) will depress the per capita growth rate, in which case different appropriate biological assumptions will be considered.

We also assume that, in the absence of Allee effects, when population size is low, resources available for individuals are sufficiently abundant for the birth rate to exceed the death rate and so $f\left(0^{+}\right)>0$. This is still the case for weak Allee effects, but, of course, it fails if there are strong Allee effects, in which case we have $f\left(0^{+}\right)<0$.

We will now incorporate the effect of the random environmental fluctuations on the per capita growth rate (environmental stochasticity), assuming they can be approximated by a white noise of the form $\sigma \varepsilon(t)$, where $\varepsilon(t)$ is the standard white noise and $\sigma>0$ is a parameter measuring the intensity of the fluctuations.

We obtain the stochastic differential equation (SDE) general model

$$
\frac{1}{X(t)} \frac{d X(t)}{d t}=f(X(t))+\sigma \varepsilon(t), \quad X(0)=x>0 .
$$

Since the accumulated noise up to time $t$ is given by $\sigma W(t)$, where $W(t)=$

${ }_{60} \int_{0}^{t} \varepsilon(s) d s$ is the standard Wiener process, it can be written in the standard format

$$
d X(t)=f(X(t)) X(t) d t+\sigma X(t) d W(t), \quad X(0)=x>0,
$$


where we take $f$ as being the geometric average per capita growth rate (working with geometric averages is more natural since we are dealing with a growth process). Having chosen the geometric average, we should and will use Stratonovich calculus in the study of the SDE (3). If one prefers to use Itô calculus, only slight adaptations to our treatment are required and one should bear in mind that, for Itô calculus, $f$ represents the arithmetic average per capita growth rate; the results are identical if one takes into account the difference between the geometric and the arithmetic averages. More details on this issue can be found in 5 .

Notice that the deterministic model (1) corresponds to $\sigma=0$.

In Section 2 we look at some general information useful in the study of our stochastic models.

The study of general SDE population models without Allee effects can be 75 found in [5], 6]. Section 3 gives a brief account of the main results concerning existence and uniqueness of the solution, extinction and stationary behaviour of the population.

Section 4 considers similar issues for the strong Alee effects case and compares the results with the corresponding results of the model without Allee effects. Section 5 will consider the case of weak Allee effects and compares results with the model without Allee effects and with the model with strong Allee effects. Preliminary results on specific and the general Allee effects models can be found in [7] for the strong case and in [8] for the weak case. Stochastic models with Allee effects, using specific growth functions $f$ or looking at demographic stochasticity (effect of sampling variations on births and deaths, a phenomenon quite different from environmental stochasticity, which is the object of this paper) were studied, for instance, in [3, [9] and [10].

In Section 6, we illustrate the results with an example, namely a SDE logisticlike weak Allee effects model. We chose a parametrization of $f$ slightly different 90 from the one commonly used in order to make model comparisons meaningful. Section 7 presents the conclusions. 


\section{General treatment of SDE models}

For the autonomous SDE models of the form (3) (see, for instance, [1]), the diffusion coefficient $b(x)$ (also known as the infinitesimal variance since it is the of the stochastic term

$$
b(X)=\sigma^{2} X^{2}
$$

and the drift coefficient $a(x)$ (also known as infinitesimal mean since it is the speed at which the mean changes) is

$$
a(X)=X\left(f(X)+\frac{\sigma^{2}}{2}\right),
$$

which equals the deterministic term plus a correction term (due to the use of the Stratonovich calculus) $\frac{1}{4} \frac{d b(X)}{d X}=\frac{\sigma^{2}}{2} X$.

The state space for models of type (3) should be $(0,+\infty)$, with boundaries $X=0$ and $X=+\infty$. Since $a(X)$ and $b(X)$ are both of class $C^{1}$, the solution $X(t)$ exists and is unique up to a possible explosion time and is a homogeneous diffusion process with drift coefficient $a(X)$ and diffusion coefficient $b(X)$ (see, boundaries are unattainable (not reachable in finite time), so that, if one starts with an initial positive population size $X(0)=x>0$, the solution $X(t)$ of the SDE remains in the state space for all time $t \geq 0$, as it is required for a proper model of population size. In particular, explosions (i.e. solutions that reach the value $+\infty$ in finite time) are not possible, which guarantees the existence and uniqueness of the solutions for all $t \geq 0$.

To study the qualitative behaviour of population size, it is important to examine the behaviour of the two boundaries in terms of being attractive or non-attractive. When the population size reaches the vicinity of a boundary, there is a tendency to move closer to the boundary if it is attractive and to move away if it is non-attractive. Formal definitions can be found, for instance, in [11. Non-attractive boundaries cannot be reached in finite time (so they are unattainable) nor in infinite time. The behaviour of the boundary 0 is particularly important to study extinction. 
A good way of determining whether a boundary is attractive or non-attractive is to look at the behaviour of the scale measure in a neighbourhood of the boundary. The scale measure of $X$ is defined on $(0,+\infty)$ by the scale density (see, for instance, [1])

$$
s(y):=\exp \left(-\int_{n}^{y} \frac{2 a(\theta)}{b(\theta)} d \theta\right)=\frac{n}{y} \exp \left(-\frac{2}{\sigma^{2}} \int_{n}^{y} \frac{f(\theta)}{\theta} d \theta\right),
$$

where $n$ is an arbitrary (but fixed) point in the interior of the state space. The corresponding "distribution" function is the scale function $S(z)=\int_{c}^{z} s(y) d y$, where $c$ is an arbitrary (but fixed) point in the interior of the state space. The scale measures is defined for intervals $(a, b)$ by $S(a, b)=S(b)-S(a)$.

When both boundaries are non-attractive, we may have a kind of asymptotic stochastic equilibrium in the sense that the probability distribution of the population size converges, as $t \rightarrow+\infty$, to an equilibrium distribution having a probability density function (p.d.f.) $p(y)$, the so-called stationary density.

To determine whether this is the case or not, we will use the speed measure of $X$, which is defined on the interior of the state space by the speed density (see, for instance, [11])

$$
m(y):=\frac{1}{b(y) s(y)}=\frac{1}{n \sigma^{2} y} \exp \left(\frac{2}{\sigma^{2}} \int_{n}^{y} \frac{f(\theta)}{\theta} d \theta\right) .
$$

The corresponding "distribution" function is the speed function $M(z)=\int_{c}^{z} m(y) d y$. The speed measure of an interval $(a, b)$ is $M(a, b)=M(b)-M(a)$.

The speed measure has nothing to do with speed, but rather is proportional to the occupation time of the trajectories. I.e., the speed measure of a Borel set of population sizes $C$ is proportional to the time population trajectories spend taking values in $C$. Should the speed measure be finite, this is proportional to the equilibrium probability (i.e. the long-term probability as $t \rightarrow+\infty$ ) of the population size being in $C$ and the p.d.f. of such probability distribution, the stationary density $p(y)$, is proportional to the speed density $m(y)$. If the speed measure is not finite, there is no stationary density. 


\section{General stochastic model without Allee effects}

We will now consider the general model [model (1) for the deterministic situation $\sigma=0$ or model (3) for the stochastic situation $\sigma>0$ ] for the case where Allee effects are absent.

For this case, we assume that $f(X)$ is a strictly decreasing function and

either, but the situation is different for the stochastic case.

For the stochastic models, one can see (for instance, in [11]) that a boundary is attractive or non-attractive according to whether the scale measure $S$ of a 
small neighbourhood of the boundary is finite or not.

The study of the general SDE population models without Allee effects can be found in $[5$, and $[6]$.

In this case, it is shown there that $S\left(0, x_{0}\right)=\int_{0}^{x_{0}} s(y) d y=+\infty$ for $x_{0}$ in the interior of the state space. Therefore, the boundary $X=0$ is non-attracting, which implies that "mathematical" extinction has zero probability of occurring since the boundary cannot be reached in finite or infinite time (see [1] ).

It is also shown that $S\left(x_{0},+\infty\right)=\int_{x_{0}}^{+\infty} s(y) d y=+\infty$ for $x_{0}$ in the interior of the state space. Therefore, the boundary $X=+\infty$ is also non-attracting, which implies that explosions have zero probability of occurring.

Since both boundaries are non-attracting, and so unattainable, the solution exists and is unique for all $t \geq 0$, and stays in the state space $(0,+\infty)$.

We have seen that, in the deterministic case, the population size settles down as $t \rightarrow+\infty$ to a non-extinction equilibrium value $K>0$ such that $f(K)=0$. The same does not happen in the stochastic case since the random environmental fluctuations will keep the population size fluctuating. However, the p.d.f. $p(t, y)$ of $X(t)$ may settle down as $t \rightarrow+\infty$ to an equilibrium p.d.f. $p(y)$, the so-called stationary density. So, we may have a kind of stochastic equilibrium instead of a deterministic one.

One can see (for instance in [1]) that, when both boundaries are nonattractive, the stationary density exists if and only if the speed measure is finite, which means that the speed density is integrable in the state space:

$$
M=\int_{0}^{+\infty} m(z) d z<+\infty .
$$

If that happens, the stationary density is given by

$$
p(y)=\frac{m(y)}{M}, 0<y<+\infty
$$

and, moreover, $X(t)$ is an ergodic process. This implies that $X(t)$ will reach any point in the interior of the state space infinitely often. Further, for large $t$ and any Borel set $C \subset(0,+\infty)$, the probability that $X(t) \in C$ is approximately $\int_{C} p(y) d y$. 
It can be found in [5], 6] that, for the general SDE model without Allee effects, we indeed have $M<+\infty$.

Taking into account (7), (8) and (9), the stationary density is given by

$$
p(y)=\frac{1}{D} \frac{1}{\sigma^{2} y} \exp \left(\frac{2}{\sigma^{2}} \int_{n}^{y} \frac{f(\theta)}{\theta} d \theta\right) \quad(0<y<+\infty),
$$

205 large $K$ shows that the population will randomly fluctuate in a neighbourhood of $K$ for long periods, after which it leaves that region to visit other regions of the state space and later come back to the neighbourhood of $K$. Such visits will

Consider the case of small $\sigma$. We have $f(K)=0$ and $f$ is strictly decreasing, so $d f(y) / d y$ is negative in the neighbourhood of $K$. Therefore, for small enough $\sigma$, there is one and only one solution $y=B$ of $f(y)=\sigma^{2} / 2$ and that solution should be close to $K$, but smaller than $K$. Furthermore, $d p(B) / d y=0$ and $d^{2} p(B) / d y^{2}<0$ (easy computations show that $d^{2} p(B) / d y^{2}$ has the same sign as $B d f(B) / d y$, which is negative). Therefore, $B$ is a maximum of the stationary density, i.e. a mode of the stationary distribution. So, when the intensity of the environmental fluctuations is small, the stationary distribution has a single mode, which is smaller than the carrying capacity but very close to it.

In conclusion, for the general SDE model without Allee effects, the solution exists and is unique, "mathematical" extinction has zero probability of occurring and there is a stochastic equilibrium with stationary density given by $(10$, , 11). We also conclude that the process is ergodic.

We end this section with a final comment on extinction. For the deterministic model, both "mathematical" and "realistic" extinction cannot occur. However, in the stochastic case, although "mathematical" extinction has a zero probability of occurring, "realistic" extinction occurs with probability one because the process is ergodic and will, sooner or later, cross the "realistic" extinction threshold $a$.

Under the stationary regimen (i.e., for large $t$ ), a large deviation argument for 
sometimes be to a neighbourhood of zero but the non-attractivity of the zero

illustration). The equilibrium $X=A$ is unstable and the equilibria $X=0$ (extinction) and $X=K$ are stable. So, if the initial population size is below $A$, the population will become extinct since $X(t) \rightarrow 0$ as $t \rightarrow+\infty$. However, if the initial population size is above $A$, then the population size will approach $K$ 255 since $X(t) \rightarrow K$ as $t \rightarrow+\infty$.

For the stochastic model, the boundary $X=0$ is attracting but unattainable, so there is no finite $t$ such that $X(t)=0$; however, it may happen that 
$X(t) \rightarrow 0$ when $t \rightarrow+\infty$ and so "mathematical" extinction can occur in infinite time. In fact, that will happen with probability one since the other boundary is non-attracting and any small neighbourhood of the attracting boundary zero is reachable with positive probability.

To show that $X=0$ is an attracting boundary, it suffices to notice that, for $0<x_{0}<n<A$ and $0<y \leq x_{0}$, we have, with $h(y)=-\frac{2}{\sigma^{2}} \int_{n}^{y} \frac{f(\theta)}{\theta} d \theta$ and noticing that $h\left(0^{+}\right)<0$,

$$
\begin{aligned}
S\left(0, x_{0}\right] & =\int_{0}^{x_{0}} \frac{n}{y} \exp \left(-\frac{2}{\sigma^{2}} \int_{n}^{y} \frac{f(\theta)}{\theta} d \theta\right) d y \\
& \leq \int_{0}^{x_{0}}-\frac{\sigma^{2} n}{2 f\left(x_{0}\right)} \frac{d\left(\exp \left(-\frac{2}{\sigma^{2}} \int_{n}^{y} \frac{f(\theta)}{\theta} d \theta\right)\right)}{d y} d y \\
& =c_{1}\left(\exp \left(h\left(x_{0}\right)\right)-\exp \left(h\left(0^{+}\right)\right)\right)<+\infty,
\end{aligned}
$$

where $c_{1}>0$ is a constant.

For the stochastic models, one can see (for instance, in [11]) that a boundary is attainable or unattainable according to whether $\Sigma\left(0, x_{0}\right]=\int_{0}^{x_{0}} S(0, z) m(z) d z$ is finite or not. In this case, we have

$$
\begin{aligned}
\Sigma\left(0, x_{0}\right] & =\frac{1}{\sigma^{2}} \int_{0}^{x_{0}} \frac{1}{z} \int_{0}^{z} \frac{1}{y} \exp \left(\frac{2}{\sigma^{2}} \int_{y}^{z} \frac{f(\theta)}{\theta} d \theta\right) d y d z \\
& \geq \frac{1}{\sigma^{2}} \int_{0}^{x_{0}} \frac{1}{z} \int_{0}^{z} \frac{1}{y} \exp \left(\frac{2}{\sigma^{2}} \int_{y}^{z} \frac{f(y)}{\theta} d \theta\right) d y d z \\
& =\frac{1}{\sigma^{2}} \int_{0}^{x_{0}} \frac{1}{z} \int_{0}^{z} \frac{1}{y}\left(\frac{z}{y}\right)^{\frac{2}{\sigma^{2}} f(y)} d y d z \\
& =\frac{1}{\sigma^{2}} \int_{0}^{x_{0}} y^{-\frac{2}{\sigma^{2}} f(y)-1} \int_{y}^{x_{0}} z^{\frac{2}{\sigma^{2}} f(y)-1} d z d y \\
& =\int_{0}^{x_{0}} \frac{1-\left(\frac{y}{x_{0}}\right)^{\frac{2}{\sigma^{2}}|f(y)|}}{2 y|f(y)|} d y .
\end{aligned}
$$

Since we have assumed $f\left(0^{+}\right)$finite, for $x_{0}$ small enough we have $f\left(0^{+}\right)-\epsilon<$ $f(y)<f\left(0^{+}\right)+\epsilon$, with $\epsilon<\frac{\left|f\left(0^{+}\right)\right|}{2}$. So, $\left|f\left(0^{+}\right)\right|-\epsilon<|f(y)|<\left|f\left(0^{+}\right)\right|+\epsilon$.

Therefore,

$$
\Sigma\left(0, x_{0}\right] \quad \geq \int_{0}^{x_{0}} \frac{1-\left(\frac{y}{x_{0}}\right)^{\frac{2}{\sigma^{2}}\left(\left|f\left(0^{+}\right)\right|-\epsilon\right)}}{2 y\left(\left|f\left(0^{+}\right)\right|+\epsilon\right)} d y
$$




$$
=\frac{\int_{0}^{x_{0}} \frac{1}{y} d y}{2\left(\left|f\left(0^{+}\right)\right|+\epsilon\right)}-\frac{\int_{0}^{x_{0}} y^{\frac{2}{\sigma^{2}}\left(\left|f\left(0^{+}\right)\right|-\epsilon\right)-1}}{2\left(\left|f\left(0^{+}\right)\right|+\epsilon\right) x_{0}^{\frac{2}{\sigma^{2}}\left(\left|f\left(0^{+}\right)\right|-\epsilon\right)}} d y=+\infty,
$$

since the first integral is divergent with value $+\infty$ and the second integral is convergent. This shows that $X=0$ is an unattainable boundary.

For the stochastic model, the boundary $X=+\infty$ is non-attracting. This is indeed the case, since, for $0<n<x_{0}$ and $K<x_{0} \leq y<+\infty$, we have

$$
S\left[x_{0},+\infty\right) \geq \exp \left(-\frac{2}{\sigma^{2}} \int_{n}^{x_{0}} \frac{f(\theta)}{\theta} d \theta\right) \int_{x_{0}}^{+\infty} \frac{n}{y} d y=+\infty .
$$

Since both boundaries are unattainable, the solution exists and is unique for all $t \geq 0$, and stays in the state space $(0,+\infty)$.

The stationary density $p(y)$, when it exists, must satisfy the Kolmogorov forward equation

$$
\frac{d(a(y) p(y))}{d y}-\frac{1}{2} \frac{d^{2}(b(y) p(y))}{d^{2} y}=0,
$$

We can show that every non-negative solution is not integrable $\left(\int_{0}^{+\infty} p(y) d y=+\infty\right)$ and, consequently, contrary to the corresponding stochastic model without Allee effects, there is no stationary density.

The solution of the differential equation 12 is

$$
p(y)=m(y)(C S(y)+D) .
$$

We can write

$$
p(y)=m(y)\left(C S(0, y)+D^{*}\right),
$$

with $D^{*}=D+C S(0)$ constant (notice that $S(0)$ is finite since $X=0$ is attractive).

Since the $X=0$ boundary is unattainable, we have

$$
\int_{0}^{+\infty} S(0, z) m(z) d z=\Sigma\left(0, x_{0}\right]+\int_{x_{0}}^{+\infty} S(0, z) m(z) d z=+\infty,
$$

because the first integral is divergent and the integrand in the second integral is positive.

We now show that $M=\int_{0}^{+\infty} m(y) d y=+\infty$. Let $0<y_{1}<A<K<+\infty$ and $y_{1}<n$. Consider the case $n<A<K$; the proof in the other case is similar 
and so will not be shown here. Let us break the integration interval:

$$
M=M_{1}+M_{2}=\int_{0}^{y_{1}} m(y) d y+\int_{y_{1}}^{+\infty} m(y) d y .
$$

We first show that $M_{1}$ is infinite. If $y \in\left(0, y_{1}\right]$ and $\theta \in[y, n]$, then

$$
\begin{aligned}
M_{1} & \geq \int_{0}^{y_{1}} \frac{1}{n \sigma^{2} y} \exp \left(-\frac{2}{\sigma^{2}} f(n) \int_{y}^{n} \frac{1}{\theta} d \theta\right) d y \\
& =c_{1} \int_{0}^{y_{1}} y^{\frac{2}{\sigma^{2}} f(n)-1} d y=+\infty,
\end{aligned}
$$

with $c_{1}>0$ constant, because $n<A$ and so $f(n)<0$. Since $M_{2}$ is non-negative

290 because its integrand is non-negative, we conclude that $M=+\infty$.

If $C=0$ and $D^{*} \neq 0$, since $\int_{0}^{+\infty} m(y) d y=+\infty$, we have $\int_{0}^{+\infty} p(y) d y=+\infty$. If $C \neq 0$ and $D^{*}=0$, since $\int_{0}^{+\infty} m(y) S(0, y) d y=+\infty$, we have $\int_{0}^{+\infty} p(y) d y=$ $+\infty$. If $C=0$ and $D^{*}=0$, then $\int_{0}^{+\infty} p(y) d y=0$. Therefore, for $p(y)$ to be a probability density, it is necessary that $C$ and $D^{*}$ are both non-zero.

In order to get $p(y) \geq 0$, as required to be a probability density, we need $C S(0, y)+D^{*} \geq 0$ for all $y \geq 0$, and since $S(0,0)=0$, we need $D^{*} \geq 0$; however, we have shown that $D^{*}=0$ is not compatible with $p(y)$ being a probability density. So we conclude that, for $p(y)$ to be a probability density, it is necessary that $D^{*}>0$.

As $S(0,+\infty)=+\infty$, if $C<0, p(y)$ would have negative values for some values of $y$. Therefore, in order for $p(y)$ to be a probability density, we must have $C>0$. So, we must have both $C>0$ and $D^{*}>0$. But, for $C>0$ and $D^{*}>0$, we have $\int_{0}^{+\infty} p(y) d y=+\infty$ and $p(y)$ would not be a probability density. Therefore, we conclude that there is no stationary density.

In conclusion, for the stochastic situation, "mathematical" extinction will occur with probability one and there is no stationary density, contrary to models without Allee effects. As for the deterministic situation, the limiting behaviour also differs from models without Allee effects, for which the population size always approaches the carrying capacity; with strong Allee effects, the limiting behaviour depends on the initial condition and the population will become extinct or will converge to the carrying capacity according to whether the initial 
population size is below or above $A$. Notice that existence and uniqueness of solutions are insured with the mild assumptions on $f$ that we have made.

\section{General stochastic model with weak Allee effects}

In this Section, we consider the case of weak Allee effects.

The asumptions on $f$ are exactly the same as in Section 4, except that now $f\left(0^{+}\right)>0$. You can see Figure 1 for illustration.

It is easy to show that $f(X)>0$ for $X<K$ and $f(X)<0$ for $X>K$. So, the only deterministic equilibria are $X=0$, which is unstable, and $X=K$, which is globally asymptotically stable. Therefore, for the deterministic model, the population size will converge to the carrying capacity $K$ as $t \rightarrow+\infty$.

For the stochastic model $(\sigma>0)$, the boundary $X=0$ is non-attracting. This is indeed the case, since, for $0<x_{0}<n$ and $0<y \leq x_{0}<L$, we have

$$
S\left(0, x_{0}\right] \geq \int_{0}^{x_{0}} \frac{n}{y} d y=+\infty .
$$

Therefore, "mathematical" extinction has zero probability of occurring.

Using an argument identical to the one used in the previous Section, one can show that the boundary $X=+\infty$ is non-attractive.

Since both boundaries are non-attracting, and so unattainable, the solution exists and is unique for all $t \geq 0$, and stays in the state space $(0,+\infty)$.

We have seen that, in the deterministic case, the population size settles down as $t \rightarrow+\infty$ to a non-extinction equilibrium value, $K>0$. We know that the same does not happen in the stochastic case since the random environmental fluctuations will keep the population size fluctuating. However, as we know, a stochastic equilibrium with a stationary distribution may be possible, in which case the process is ergodic.

We now show that this is indeed the case, by proving that $M$, given by (8), is finite.

Let $y_{1}<K<y_{2}$ be such that $0<y_{1}<n<y_{2}<+\infty$ : Assume that $n<L$ (the proof for $n>L$ is similar and will not be shown here). So $0<y_{1}<n<$ 
$L<K<y_{2}$. Let us break the integration interval as follows:

$$
M=M_{1}+M_{2}+M_{3}=\int_{0}^{y_{1}} m(y) d y+\int_{y_{1}}^{y_{2}} m(y) d y+\int_{y_{2}}^{+\infty} m(y) d y .
$$

We first show that $M_{1}$ is finite. Let $y \in\left(0, y_{1}\right]$ and $\theta \in[y, n]$. Since $0<$ $f\left(0^{+}\right)<+\infty$, we have

$$
\begin{aligned}
m(y) & =\frac{1}{n \sigma^{2} y} \exp \left(-\frac{2}{\sigma^{2}} \int_{y}^{n} \frac{f(\theta)}{\theta} d \theta\right) \\
& \leq \frac{1}{n \sigma^{2} y} \exp \left(-\frac{2}{\sigma^{2}} \int_{y}^{n} \frac{f\left(0^{+}\right)}{\theta} d \theta\right)=\frac{1}{n^{2} \sigma^{2}}\left(\frac{y}{n}\right)^{\frac{2}{\sigma^{2}} f\left(0^{+}\right)-1},
\end{aligned}
$$

because $f$ is increasing. Therefore $M_{1}<+\infty$.

We now prove that $M_{3}<+\infty$. Let $y \in\left[y_{2},+\infty\right)$ and $\theta \in[n, y]$. Decompose

$$
\frac{2}{\sigma^{2}} \int_{n}^{y} \frac{f(\theta)}{\theta} d \theta=\frac{2}{\sigma^{2}} \int_{n}^{y_{2}} \frac{f(\theta)}{\theta} d \theta+\frac{2}{\sigma^{2}} \int_{y_{2}}^{y} \frac{f(\theta)}{\theta} d \theta=B+C(y) .
$$

Then

$$
m(y) \leq \frac{1}{n \sigma^{2} y} \exp (B) \exp \left(\frac{2}{\sigma^{2}} f\left(y_{2}\right) \ln \frac{y}{y_{2}}\right) .
$$

Therefore, since $K<y_{2}$ and $f\left(y_{2}\right)<0$, we get

$$
M_{3} \leq c_{1} \int_{y_{2}}^{+\infty} y^{\frac{2}{\sigma^{2}} f\left(y_{2}\right)-1} d y<+\infty
$$

with $c_{1}$ constant.

Finally, it is easy to see that $M_{2}<+\infty$ because it is the integral of a continuous function in a closed interval.

The stationary density is given by 10 and (11). Like in the models without Allee effects, for a small environmental noise intensity $\sigma>0$, the stationary density has a mode $B<K$ close to $K$, which is the solution of $f(B)=\sigma^{2} / 2$.

We conclude that the weak Allee effects models have the same qualitative behaviour as the models with no Allee effects.

Weak Allee effects models have, however, a qualitative behaviour quite different from the strong Allee effects models. In the deterministic situation, we have convergence to the carrying capacity, while in the strong Allee effects case we have a limiting behaviour that depends on the initial condition (extinction or convergence to the carrying capacity according to whether the initial population 
size is below or above $A$ ). In the stochastic situation, there is no mathematical extinction and there is a stochastic equilibrium with a stationary density, while in the strong Allee effects case "mathematical" extinction will occur with probability one and there is no stationary density.

In terms of the population size at any time $t>0$, assuming the same initial condition $X(0)=x>0$, the same carrying capacity, the same environmental conditions (i.e., the same random trajectory of the Wiener process $W(t)$ ), and everything else equal, what would happen if we compare three different scenarios for the population, one without Allee effects, the second with weak Allee effects and the third with strong Allee effects? Of course, in this hypothetical experiment, Allee effects would depress the per capita growth rate at low population densities, more so if they were strong effects. So, we could assume that the per capita growth rates for the three scenarios, respectively $f, f_{w}$ and $f_{s}$, satisfy the relation $f(X) \geq f_{w}(X) \geq f_{s}(X)$ with strict inequalities at least for low population sizes $X$. The comparison theorems for SDE (see [16] and [17]) show that the population size would be the largest in the first scenario and the smallest in the third scenario, with the population of the second scenario in between. The same is true for the deterministic models. So, as should be expected, Allee effects have consequences on population sizes, depressing their values compared to the case where Allee effects are absent.

\section{SDE logistic-like Allee effects model}

As an illustration, we will consider the particular case of a logistic-like Allee effect model that has been considered in the deterministic literature, where $f$ is now a second-degree polynomial. However, we present the model with a 375 slightly different parametrization in order to allow comparisons with the classical logistic model without Allee effects. Notice that there are no substantive reasons to believe this model is an appropriate description of population growth under Allee effects, and that is why we rather worked before with very general models in order to obtain properties that are model-robust. 
The classical logistic model with no Allee effects corresponds to

$$
f(X)=r\left(1-\frac{X}{K}\right)
$$

with $r>0$ (intrinsic growth rate) and $K>0$.

We are going to use a logistic-like model with Allee effects having the same carrying capacity $K$ and with $f(X)$ having the same slope $-r / K$ when $X=K$. This model has the form

$$
f(X)=r\left(1-\frac{X}{K}\right) \frac{X-A}{K-A},
$$

with an extra parameter $A$ such that $A \neq 0$ (to satisfy the condition that $\left.f\left(0^{+}\right) \neq 0\right)$.

In order to have Allee effects, we need $f$ to have a maximum at a point $L$ with $0<L<K$, so that $f(X)$ will strictly increase for $X<L$ and strictly decrease for $X>L$. To achieve that, we need to assume that $-K<A<K$ and, with that assumption, $L=(K+A) / 2$.

When $0<A<K$, we have $f\left(0^{+}\right)=-r A /(K-A)<0$ and a strong Allee effects. In this case, the parameter $A$ is precisely the value in the $(0, L)$ interval for which $f(A)=0$ mentioned in Section 4. In Figure 1, the dashed line corresponds to the value $A=0.15 K$ (labelled on the Figure) and $L$ is labelled as $L_{\text {strong. }}$

When $-K<A<0$, we have $f\left(0^{+}\right)=-r A /(K-A)>0$ and a weak Allee effect. In Figure 1, the dotted line corresponds to the value $A=-0.15 K$ (not labelled on the Figure) and $L$ is labelled as $L_{\text {weak }}$.

Actually, $A$ is a measure of the strength of the Allee effects; the larger it is, the stronger are the Allee effects.

If we would consider values $A>K$, the model would be nonsensical since $f$ would take positive values for very large population sizes. The case $A=K$ is obviously not possible. So, we exclude such possibilities.

However, we can consider values of $A \leq-K$. The only problem is that they are not of the Allee effects type, since $f$ will not have a maximum $L$ in the interval $(0, K)$; of course, if we use as a basis for comparison the logistic 
model without Allee effects, a model with $A \leq-K$ will have a smaller per capita growth rate at low population densities, but not small enough to be classified as an Allee effects model.

It is quite interesting that, when $A \rightarrow-\infty$, we retrieve the classical logistic model without Allee effects (16). This allows us to compare the logistic-like models with Allee effects, either weak (for $-K<A<0$ ) or strong (for $0<$ $A<K)$, among them and with the classical logistic model without Allee effects $(A=-\infty)$.

The qualitative behaviour of the deterministic and stochastic models in this particular case of $f$ given by (17) is as described in the previous Sections since $f$ satisfies their assumptions. Therefore existence and uniqueness of the solutions are insured and we have:

- Case $A<0$, which includes the logistic model $(A=-\infty)$ and weak Allee effects models $(-K<A<0)$. For the deterministic model, there is no "mathematical" extinction, $K$ is a globally asymptotically stable equilibrium and $X(t) \rightarrow K$ as $t \rightarrow+\infty$. For the stochastic model, the boundary $X=0$ is non-attracting, "mathematical" extinction has zero probability of occurring, there is a stochastic equilibrium with a stationary density (we will give below its expression for this particular case) and the process is ergodic.

- Case $0<A<K$, which corresponds to strong Allee effects. For the deterministic model, there is an unstable equilibrium $A$ and the two other equilibria, $X=0$ and $X=K$, are stable. The population will become extinct when the initial size $x<A$ and will converge to $K$ when the initial size $x>A$. For the stochastic model, the boundary $X=0$ is attracting (but unattainable in finite time), "mathematical" extinction will occur with probability one and there is no stationary density.

We remind that, for the stochastic models with finite $A<K$, the solution 435 $X(t)$ is a homogeneous diffusion process and, for this particular case, the drift 
coefficient is

$$
a(X)=\left(r\left(1-\frac{X}{K}\right) \frac{X-A}{K-A}+\frac{\sigma^{2}}{2}\right) X,
$$

and the diffusion coefficient is

$$
b^{2}(X)=\sigma^{2} X^{2} .
$$

From (6) and (7), using (17), we obtain, after some algebra, the scale density

$$
s(y)=C y^{\frac{2 r A}{\sigma^{2}(K-A)}-1} \exp \left(\frac{r}{\sigma^{2} K(K-A)}(y-(K+A))^{2}\right)
$$

and the speed density

$$
m(y)=\frac{1}{C \sigma^{2}} y^{-\frac{2 r A}{\sigma^{2}(K-A)}-1} \exp \left(-\frac{r}{\sigma^{2} K(K-A)}(y-(K+A))^{2}\right),
$$

with $C>0$ constant.

Of course, when $A<0$ finite, the speed density is integrable and the stationary density exists and is given by

$$
p(y)=\frac{1}{D \sigma^{2}} y^{-\frac{2 r A}{\sigma^{2}(K-A)}-1} \exp \left(-\frac{r}{\sigma^{2} K(K-A)}(y-(K+A))^{2}\right) \quad(0<y<+\infty),
$$

with

$$
D=\int_{0}^{+\infty} \frac{1}{\sigma^{2}} y^{-\frac{2 r A}{\sigma^{2}(K-A)}-1} \exp \left(-\frac{r}{\sigma^{2} K(K-A)}(y-(K+A))^{2}\right) d y .
$$

When $0<A<K$, the speed density is not integrable and there is no stationary 445 density.

For the logistic model given by (16) (corresponding to $A=-\infty$ ), we have

$$
\begin{gathered}
a(X)=\left(r\left(1-\frac{X}{K}\right)+\frac{\sigma^{2}}{2}\right) X \\
b(X)=\sigma^{2} X^{2} \\
s(y)=D y^{-\frac{2 r}{\sigma^{2}}-1} \exp \left(\frac{2 r}{\sigma^{2} K} y\right) \quad(0<y<+\infty) \\
m(y)=\frac{1}{C \sigma^{2}} y^{\frac{2 r}{\sigma^{2}}-1} \exp \left(-\frac{2 r}{\sigma^{2} K} y\right),
\end{gathered}
$$


In the case of weak Allee effects and in the case of absence of Allee effects, "mathematical" extinction does not occur, both for the deterministic models $(\sigma=0)$ and for the stochastic models $(\sigma>0)$. For the deterministic models, 470 carrying capacity $K$. The same does not happen for the stochastic models, since the random environmental fluctuations will make the population size fluctuate as well; however, we have shown that there is a stochastic equilibrium with a stationary density $p(y)(0<y<+\infty)$, which we have determined. 
In the case of strong Allee effects,"mathematical" extinction occurs with probability one for the stochastic model and no stationary density exists. For the deterministic model, we can have two possible outcomes, extinction or convergence to the carrying capacity, depending on whether the initial population size is below or above a certain value $A$.

We have illustrated the results with an example, namely a SDE logistic-like Allee effects model.

We have proved existence of a stationary density and a zero probability of "mathematical" extinction for the general stochastic models with weak Allee effects model and without Allee effects. However, as we have shown, "realistic" extinction will occur with probability one for all our models. So, it is important to study the time for "realistic" extinction of the population. We will do that in a further paper, as well as compare the extinction times for the SDE models without Allee effects, with strong Allee effects and with weak Allee effects.

\section{Acknowledgements}

Both authors belong to the Centro de Investigação em Matemática e Aplicações, Universidade de Évora, a research centre supported by FCT (Fundação para a Ciência e a Tecnologia, Portugal), project UID/MAT/04674/2013.

\section{References}

[1] W. Allee, A. Emerson, O. Park, T. Park, K. Schmidt, Principles of Animal Ecology, Saunders, Philadelphia, 1949.

[2] F. Courchamp, T. Cliutton-Brock, B. Grenffell, Inverse density dependence and the Allee effect, Trends in Ecology \& Evolution 14 (1999) 405-410.

[3] B. Dennis, Allee effects: Population growth, critical density and the chance of extinction, Natural Resource Modelling 3 (1989) 481-539.

500

[4] M. Lewis, P. Kareiva, Allee dynamics and the spread of invading organisms, Theoretical Population Biology 43 (1993) 141-158. 
[5] C. Braumann, Itô versus stratonovich calculus in random population growth, Mathematical Biosciences 206 (2007) 81-107.

[6] C. Braumann, Growth and extinction of populations in randomly varying environments, Computers and Mathematics with Applications 56 (2008) 631-644.

[7] C. Braumann, C. Carlos, Allee effects in randomly varying environments, in: Proceedings 2013 International Conference on Computational and Mathematical Methods in Science and Engineering, 2013, pp. 304-307.

[8] C. Braumann, C. Carlos, Weak allee effects population growth models in a random environment, in: Proceedings 60th ISI World Statistics Congress, 2015, pp. 2455-2460.

[9] B. Dennis, Allee effects in stochastic populations, Oikos 96 (2002) 389-401.

[10] S. Engen, R. Lande, B.-E. Sæther, Demographic stochasticity and Allee effects in populations with two sexes, Ecology 84 (9) (2003) 2378-2386.

[11] S. Karlin, H. Taylor, A Second Course in Stochastic Processes, Academic Press, New York, 1981.

[12] L. Arnold, Stochastic Differential Equations: Theory and Applications, John Wiley \& Sons, New York, 1974.

[13] C. Braumann, P. Filipe, C. Carlos, C. Roquete, Growth of individuals in randomly fluctuating environments, in: Proceedings of the International Conference in Computational and Mathematical Methods in Science e Engineering, 2009, pp. 201-212.

[14] C. Carlos, C. Braumann, P. Filipe, Models of individual growth in a ran525 dom environment: study and application of first passage times, Advances in Regression, Survival Analysis, Extreme Values, Markov Processes and Other Statistical Applications (2013) 103-111. 
[15] P. Filipe, C. Braumann, C. Carlos, Profit optimization for cattle growing in a randomly fluctuating environment, Optimization 64 (6) (2015) 13931407.

[16] S. Peng, X. Zhu, Necessary and sufficient condition for comparison theorem for 1-dimensional stochastic differential equations, Stochastic Proc. Appl. 116 (2006) 370-380.

[17] H. Zhiyuan, A comparison theorem for solutions of stochastic differential equations and its applications, Proc. Amer. Math. Soc. 91 (1984) 611-617. 